\title{
Critics condemn NIH women's study
}

\author{
Nobody disputes that women have health problems that differ from those of men, but NIH may be wasting \\ $\$ 625$ million or more in pursuit of an ambition to be seen to be politically correct.
}

WHEN the US National Institutes of Health (NIH) embarked two years ago on a \$625million 14-year study of women's health, they were able for a time to bask in approval (or self-approval) for having done the "right thing". But now the Women's Health Initiative (WHI) has been severely criticized by a committee of the Institute of Medicine (IOM), itself appointed in response to a request by the Congress for an appraisal of one of NIH's most expensive programmes ever. The question now is what went wrong.

By any standards, WHI is a massive epidemiological trial. It is one of the largest and longest ever undertaken by the NIH. Eventually, some 160,000 post-menopausal women will have been asked to take part. Among the objectives are to tell whether low-fat diets will prevent breast cancer, whether hormone replacement therapy will reduce the incidence of coronary heart disease among women and whether calcium and/or vitamin D supplements will reduce the incidence of osteoporosis. The study was put together with unusual speed in response to the determination of Bernardine Healy, the former director of NIH, to join the women's health bandwagon.

The committee's appraisal of the study, published earlier this week, is a scathing indictment of its conception and design. The head of the committee, Marion J. Finkel of the Sandoz Pharmaceutical Corporation, was chosen specifically because she is in no way reliant on NIH grants. Among public documents of this kind, the committee's report stands out for its candour. The preface, for example, says: "Had this committee been asked to design a plan for women's health research, it would not have designed this Women's Health Initiative."

The committee criticizes the WHI on several grounds, including the following: (1) The study is based on premises that are scientifically weak; (2) it is unlikely to provide statistically valid answers to the questions it asks, partly because its statistical design is poor and partly because the questions it asks are scientifically limited; (3) the study's informed consent procedures do not adequately inform women of what they are getting into; (4) the \$625-million price, high as it is, probably underestimates the true cost.

The report makes clear the misgivings of the committee about the WHI. Thus the committee attributes the request for an evaluation of WHI to "nagging doubts ... that political concerns influenced the design, timetable and budget" on the part of the
House of Representatives Appropriations Committee. Bluntly, the House asked whether the study is worth the money it will cost. In its preface, the committee frankly admits that some members would have been happy to see the WHI cancelled altogether, and that those who argued for its continuation (with significant modifications) did so primarily because the study had already begun.

A few examples will illustrate the nature of the committee's objections to the WHI protocol. Thus, on the questionable hypothesis that a reduction in dietary fat will lead to a reduced incidence of breast cancer, the committee cites a number of large-scale studies that either refute or fail to find a direct correlation (see Nature 359, 760; 1992) and goes on to challenge NIH's rosy view that WHI will produce different results, especially in a short time.

The proposed protocol assumes a linear halving of the risk over five years... the biology of breast cancer would seem to make this an optimistic projection. If diet does have an effect on breast cancer, but the lag time for halving the risk is, for example, 20 years, then the currently proposed project has very little chance to detect an effect.

Why did NIH not think of that?

The NIH protocol is also criticized for the factorial design that simultaneously assigns some participants to two arms of the trial: diet modification and hormone replacement therapy. The committee argues on practical grounds that the chances of getting a women to stick to a low-fat diet for 14 years while also staying on hormone therapy for the duration are slim. It adds that, even in the best case, the number of women in the overlap group (approximately 16 per cent) does not provide "adequate statistical power" to assess the interactions between the two regimens.

The proposals not to categorize women by known risk also provoke the committee's concern. Thus it argues that if risk factors, such as a family history of breast cancer, are not taken into account, the numbers of women in various of the study arms are too small to produce statistically meaningful data. Furthermore, the lack of information about risk factors would lead women to participate in a trial that could be hazardous to their health. There is, for example, nothing in the consent literature to warn a woman at risk from breast cancer that her risk might increase if, as one of the two-for-one participants, she agreed to take hormone therapy while also restricting dietary fat.

On osteoporosis, the critics say that the NIH protocol will not allow useful distinctions to be made between calcium, vitamin $\mathrm{D}$ and the two in combination if, indeed, the trial data show that women in the study have fewer bone fractures. Similarly, the samples required by the protocols for assessing disease risk in minority groups (post-menopausal black and Hispanic women) are said to be too small to produce meaningful data. And the litany of criticisms goes on.

How can NIH, widely regarded as the fountainhead of the good design of trials and experiments, have perpetrated such a faulty design as that of WHI? NIH's intramural scientists, who would have responded to Healy's original call, have always been well regarded. But although there is always room for disagreement about the fine points of study designs and the interpretation of the data they yield, it is unusual for a review committee (whose own work is further subjected to the review board of the National Academy of Sciences, of which the IOM is a branch) to publish such a thorough condemnation of a study designed by fellow professionals.

A majority of the committee persuaded the remainder that WHI should be allowed to continue if NIH modifies its premises and corrects its statistical procedures, but the committee also acknowledges that politics, not science, has dissuaded it from outright cancellation of the study that Healy wrought.

In reality, committee members were besieged by women's health groups who said that cancelling the study would be a sign that the NIH does not care about women. Data indicating that approximately 14 per cent of NIH money is spent on diseases that affect women, compared with 80 per cent for research on diseases that afflict women and men equally, were sidelined by NIH officials seeking arguments in favour of a conspicuous Women's Health Initiative. But they have done women a disservice by backing a hastily put-together programme that is so vulnerable to informed criticism.

The NIH leadership has not distinguished itself by swaying with the political winds over the WHI. Even worse, it has done nothing to further the entirely valid need for more well-designed, well-funded studies of diseases to which women are particularly vulnerable. But WHI is just barely under way. Is it really too late to call a halt and, with science rather than politics as the motivation, start again?

Barbara J. Culliton 